\title{
Circuit Model for a Coplanar-Slotline Cross
}

\author{
Miquel Ribó, Associate Member, IEEE, and Lluís Pradell, Member, IEEE
}

\begin{abstract}
A new 6-port "circuit-model" for Coplanar-Slotline Crosses is presented. The model is based on the separation of the two fundamental Coplanar Waveguide (CPW) modes (even and odd) into different ports. It generalizes previous models since it explains the Cross' behavior even if air-bridges are not used and Slotlines are loaded asymmetrically. The new model is applied to the analysis of the $S$-parameters of an asymmetric slotline resonator, and validated through comparison with experimental results up to 40 GHz.
\end{abstract}

Index Terms-Circuit model, coplanar waveguide, cross, resonator, slotline, transition.

\section{INTRODUCTION}

$\mathbf{T}$ RANSITIONS between Coplanar Waveguide (CPW) and Slotline are required to perform most circuit functions in planar technologies. A particular kind of transition is the CPWSlotline Cross shown in Fig. 1(a), in which a CPW is connected to two Slotlines. This transition is used in a number of circuits, such as baluns [1], slot antenna arrays [2], mixers [3], resonators [4] and slotline-coplanar series transitions [5].

In a general situation, both CPW fundamental modes, the Coplanar Even-Mode (CEM), or coplanar-mode, and the Coplanar Odd-Mode (COM), or slotline-mode, can be present at the Cross. The COM can be carried by the CPW sections from other parts of the circuit, or can be generated by asymmetries at the slotline ports. Thus, a detailed study of the CPW-Slotline Cross behavior requires a circuit-model in which both modes are considered. The Cross' models available in the literature [1]-[5] are not complete since they do not include the COM effect; to prevent its excitation either air-bridges connecting the CPW ground-planes (at the transition plane) are used [3], [5], or the Slotline ports are loaded symmetrically [2], [4].

In this letter, a new 6-port circuit-model for a CPW-Slotline Cross [Fig. 1(a)] is presented; it extends a four-port model proposed in [6] to a more general case in which slotline ports are required. In contrast to previous works [1]-[5], the model studies a general case in which the COM propagates along the CPW sections. Since it is based on the separation of modes (Slotline, CEM, and COM) into different ports, it provides a quantitative analysis of mode-conversion from Slotline mode to CEM and $\mathrm{COM}$ and vice versa, and allows the analysis of circuits in which the CPW section is loaded with structures that present different responses to each mode. Transitions with air-bridges at the CPW

Manuscript received May 25, 2000; revised October 10, 2000. This work was supported by Spanish Government Grants 2FD97-0960-C05-05 and 2FD971769-C04-03 (CICYT-FEDER), and EPSON Foundation Grant "Rosina Ribalta."

M. Ribó is with Enginyeria La Salle, Ramon Llull University (URL), Department CTS, 08022 Barcelona, Spain.

L. Pradell is with the Technical University of Catalunya (UPC), Department TSC, 08034 Barcelona, Spain.

Publisher Item Identifier S 1051-8207(00)11572-7. sections or symmetric loads at the Slotline ports [2]-[5], can also be analyzed as a particular case. Validation of the model is achieved through its application to the prediction of the $S$ parameters (from $45 \mathrm{Mhz}$ to $40 \mathrm{GHz}$ ) of an asymmetric slotline resonator.

\section{Model Derivation}

Consider the CPW-Slotline Cross shown in Fig. 1(a), in which both modes (CEM and COM) are present at the CPW sections. To derive a model, either CPW slot is seen as a transmission line propagating both, CEM and COM [see Fig. 1(b)]. The voltages and currents at the transition $\left(V_{o 1}, V_{o 2}, V_{e 1}, V_{e 2}\right.$, $V_{s a}, V_{s b}, I_{o 1}, I_{o 2}, I_{e 1}, I_{e 2}, I_{s a}$ and $\left.I_{s b}\right)$ are related by

$$
\begin{aligned}
& V_{s a}=\left(V_{e 2}-V_{e 1}\right)-\frac{V_{o 2}-V_{o 1}}{2} \\
& V_{s b}=\left(V_{e 2}-V_{e 1}\right)+\frac{V_{o 2}-V_{o 1}}{2} \\
& I_{s a}=I_{o 2}-\frac{I_{e 2}}{2}=-I_{o 1}+\frac{I_{e 1}}{2} \\
& I_{s b}=-I_{o 2}-\frac{I_{e 2}}{2}=I_{o 1}+\frac{I_{e 1}}{2}
\end{aligned}
$$

$$
\begin{array}{cl}
\text { where } & \\
\text { "e" } & \text { CEM; } \\
\text { "o" } & \text { COM; } \\
\text { "sa" } & \text { Slotline A; } \\
\text { "sb" } & \text { Slotline B. }
\end{array}
$$

From (1) and (2), the following $S$-parameters matrix is obtained for the normalized CEM, COM and Slotline incident and reflected waves at the transition, $a_{e 1}, a_{o 1}, a_{e 2}, a_{o 2}, a_{s a}, a_{s b}$, and $b_{e 1}, b_{o 1}, b_{e 2}, b_{o 2}, b_{s a}, b_{s b}$, respectively

$$
\left[\begin{array}{l}
b_{e 1} \\
b_{o 1} \\
b_{e 2} \\
b_{o 2} \\
b_{s a} \\
b_{s b}
\end{array}\right]=\frac{1}{10}\left[\begin{array}{rrrrrr}
2 & 0 & 8 & 0 & -4 & -4 \\
0 & 5 & 0 & 5 & 5 & -5 \\
8 & 0 & 2 & 0 & 4 & 4 \\
0 & 5 & 0 & 5 & -5 & 5 \\
-4 & 5 & 4 & -5 & 3 & 3 \\
-4 & -5 & 4 & 5 & 3 & 3
\end{array}\right] \cdot\left[\begin{array}{c}
a_{e 1} \\
a_{o 1} \\
a_{e 2} \\
a_{o 2} \\
a_{s a} \\
a_{s b}
\end{array}\right]
$$

where

$a_{\delta} \quad\left(V_{\delta}+Z_{0} I_{\delta}\right) \cdot\left(4 Z_{0}\right)^{-1 / 2}$

$b_{\delta} \quad\left(V_{\delta}-Z_{0} I_{\delta}\right) \cdot\left(4 Z_{0}\right)^{-1 / 2}(\delta=e 1, o 1, e 2, o 2, s a, s b)$;

$Z_{0} \quad$ real arbitrary impedance to which the normalized waves and the $6 \times 6 S$-parameter matrix are referred.

Equation (3) leads to the circuit-model proposed in Fig. 2. The four-port transition (two Slotline ports and two multi-mode CPW ports) is modeled as a 6-port device that separates the Slotline, CEM, and COM contributions into different single-mode ports: two Slotline ports (with characteristic impedances $Z_{0 s a}$ and $Z_{0 s b}$ ), two CPW ports propagating only 
the COM (with characteristic impedance $Z_{0 o}$ ), and two CPW ports propagating only the CEM (with characteristic impedance $\left.Z_{0 e}\right)$.

Since the $S$-parameters matrix in (3) does not depend on frequency, the new model is frequency-independent (provided that the exciting frequency is below the cutoff frequency of any higher-order mode excited at the transition). The model, which is easily implementable in microwave $\mathrm{CAD}$, allows a quantitative analysis of the energy transfer from one mode to the others. In particular, whenever the Slotline ports (ports 5 and 6 in Fig. 2) excite the Cross symmetrically $\left(a_{s a}=a_{s b}\right.$ ), the COM mode is not generated, as seen from (3); conversely, whenever the Slotline ports excite the Cross antisymmetrically $\left(a_{s a}=-a_{s b}\right)$, the CEM mode is not generated. Thus, by controlling the slotline excitations, the mode generated in the coplanar ports can be controlled as well. Whenever the Slotline ports are loaded symmetrically $\left(a_{s a}=a_{s b}\right)$, there is no mode conversion from CEM to COM, confirming the known fact that symmetry avoids the COM. Planar structures that present different responses to each CPW mode (such as other Slotline-to-CPW transitions and air-bridges) can be easily connected by loading the ports 1-4 in Fig. 2 with appropriate impedances.

\section{EXPERIMENTAL VALIDATION}

The proposed model has been applied to the analysis of the $S$-parameters of the asymmetric slotline resonator shown in Fig. 3(a), fabricated on Alumina $\left(\varepsilon_{r}=9.7\right.$ and thickness $0.635 \mathrm{~mm}$ ). Slotline and Coplanar slot widths are $50 \mu \mathrm{m}$ and 25 $\mu \mathrm{m}$, respectively. Coplanar central conductor width is $50 \mu \mathrm{m}$. The circuit consists of a CPW-Slotline Cross loaded with two short-circuited Slotline stubs of length $\ell_{1}=1.1 \mathrm{~mm}$, one of them short-circuited at $\ell_{2}=0.55 \mathrm{~mm}$ by an air-bridge in order to create an asymmetry). The Cross is excited by two CPW sections $\left(\ell_{3}=2 \mathrm{~mm}\right)$ propagating the CEM. Due to the asymmetry in the Slotline stubs, the COM mode is generated at the Cross and propagates along the CPW sections a distance $\ell_{4}=1$ $\mathrm{mm}$, until it is short-circuited by air-bridges. Fig. 3(a) and (b) compare its simulated $S$ parameters (obtained using the model shown in Fig. 2) with its measured ones. In the simulation, the Slotline short-circuits are modeled as small value inductors, and the air-bridges as inductors whose inductance is estimated as a function of their length and diameter. Small parasitic effects are added at the transition ports in Fig. 2 in order to take into account the reactive effect of the cut-off higher order modes generated at the transition. These parasitic effects, which cause a slight shift in the resonance frequency, are modeled as small series inductances whose values are $L_{e}=15.9 \mathrm{pH}, L_{o} \approx 0$, and $L_{s}=7.3 \mathrm{pH}$, where subindex " $e$ " refers to the CEM ports (ports 1 and 3 in Fig. 2), " $o$ " to the COM ports (ports 2 and 4 in Fig. 2), and " $s$ " to the slotline ports (ports 5 and 6 in Fig. 2). As seen in Fig. 3, the model is accurately predicting the frequency behavior of the circuit $S$-parameters, in particular its main resonance frequency at $30 \mathrm{GHz}$; the small resonance measured at $21 \mathrm{GHz}$ is attributed to a weak interaction between the COM and the wafer-probes. The good agreement between simulations and measurements demonstrates the model validity and applicability.

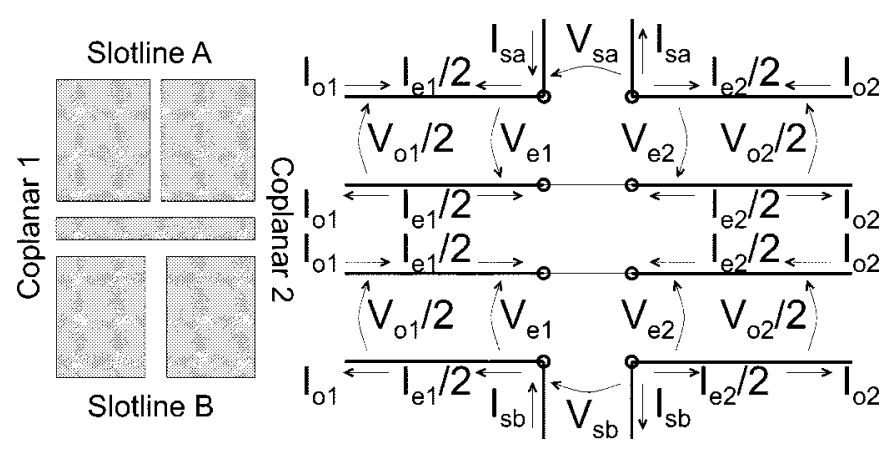

(a)

(b)

Fig. 1. Coplanar-Slotline Cross. (a) Layout. (b) Voltages and currents at the transition.

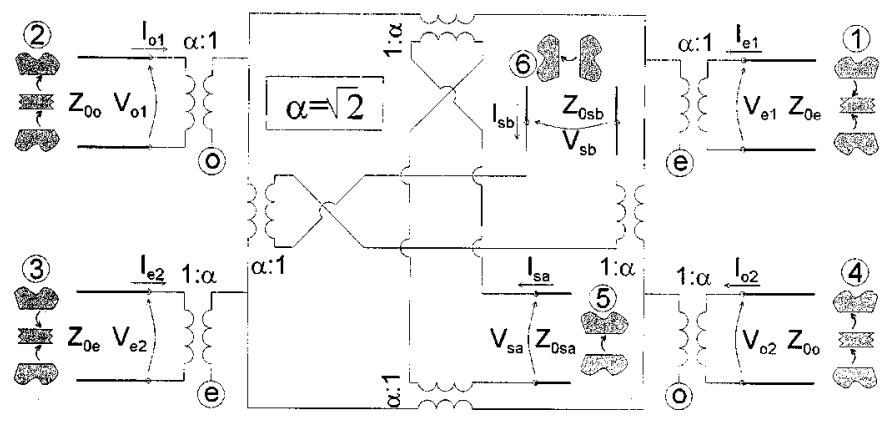

Fig. 2. Circuit-model proposed for a coplanar-slotline cross.

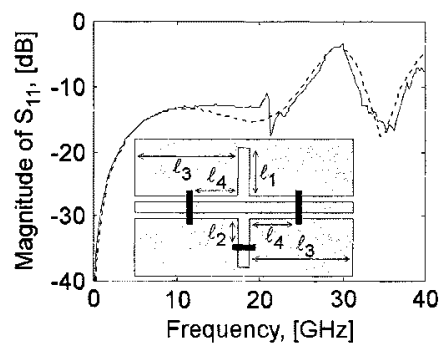

(a)

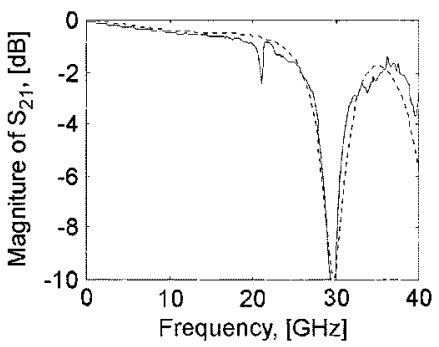

(b)
Fig. 3. Measured and simulated $S$-parameters. Measurements are shown in continuous line and simulations in dashed line. (a) Magnitude of $S_{11}$ and asymmetric slotline resonator outline. (b) Magnitude of $S_{21}$.

\section{CONCLUSIONS}

A new "circuit-model" for a CPW-Slotline Cross that separates the contributions of CPW modes into different ports, has been proposed. The model overcomes limitations of previous models, because it allows the analysis of a general case in which air-bridges are not present and slotlines are loaded asymmetrically. Validation of the model has been achieved through comparison between simulations and measurements of an asymmetric slotline resonator, showing an excellent agreement between simulation and experimental results.

\section{REFERENCES}

[1] V. Trifunovic and B. Jokanovic, "Review of printed marchand and double Y baluns: Characteristics and applications," IEEE Trans. Microwave Theory Tech, vol. 42, pp. 1154-1162, Aug. 1994.

[2] T.-F. Huang, S.-W. Lu, and P. Hsu, "Analysis and design of coplanar waveguide-fed slot antenna array," IEEE Trans. Antennas Propagat., vol. 47, pp. 1560-1565, Oct. 1999. 
[3] D. Cahana, "A new coplanar waveguide/slotline double-balanced mixer," in IEEE MTT-Symp. Dig., 1989, pp. 967-968.

[4] K. Hettak, N. Dib, A.-F. Sheta, and S. Toutain, "A class of novel uniplanar series resonators and their implementation in original applications," IEEE Trans. Microwave Theory Tech, vol. 46, pp. 1270-1276, Sept. 1998.
[5] M. Aikawa and H. Ogawa, "Double-sided MMIC's and their applications," IEEE Trans. Microwave Theory Tech, vol. 37, pp. 406-413, Feb. 1989.

[6] M. Ribó, J. de la Cruz, and L. Pradell, "Circuit model for mode conversion in coplanar waveguide asymmetric series-impedances," Electron. Lett., vol. 35, pp. 1851-1853, Oct. 1999. 